\title{
Diffusiophoresis at the macroscale
}

\author{
Cyril Mauger, ${ }^{1}$ Romain Volk, ${ }^{2}$ Nathanaël Machicoane, ${ }^{2}$ Michaël Bourgoin,,${ }^{3,2}$ \\ Cécile Cottin-Bizonne, ${ }^{4}$ Christophe Ybert, ${ }^{4}$ and Florence Raynal ${ }^{1}$ \\ ${ }^{1}$ LMFA, CNRS, Université de Lyon, École Centrale Lyon, INSA de Lyon, and \\ Université Lyon 1, 69134 Écully CEDEX, France \\ ${ }^{2}$ Laboratoire de Physique, ENS de Lyon, CNRS, and Université de Lyon, 69364 Lyon CEDEX 07, France \\ ${ }^{3}$ LEGI, CNRS, Université Joseph Fourier, Grenoble INP, 38041 Grenoble CEDEX 9, France \\ ${ }^{4}$ Institut Lumière Matière, CNRS, Université de Lyon, and Université Lyon 1, \\ 69622 Villeurbanne CEDEX, France
}

(Received 6 March 2016; published 6 July 2016)

\begin{abstract}
Diffusiophoresis, a ubiquitous phenomenon that induces particle transport whenever solute concentration gradients are present, was recently observed in the context of microsystems and shown to strongly impact colloidal transport (patterning and mixing) at such scales. In the present work we show experimentally that this nanoscale mechanism can induce changes in the macroscale mixing of colloids by chaotic advection. Rather than the decay of the standard deviation of concentration, which is a global parameter commonly employed in studies of mixing, we instead use multiscale tools adapted from studies of chaotic flows or intermittent turbulent mixing: concentration spectra and second and fourth moments of the probability density functions of scalar gradients. Not only can these tools be used in open flows, but they also allow for scale-by-scale analysis. Strikingly, diffusiophoresis is shown to affect all scales, although more particularly the small ones, resulting in a change of scalar intermittency and in an unusual scale bridging spanning more than seven orders of magnitude. By quantifying the averaged impact of diffusiophoresis on the macroscale mixing, we explain why the effects observed are consistent with the introduction of an effective Péclet number.
\end{abstract}

DOI: 10.1103/PhysRevFluids.1.034001

\section{INTRODUCTION}

Diffusiophoresis is responsible for transport of large colloidal particles under the action of solutes $[1,2]$. In the case of electrolyte (salt) concentration gradients, as will be considered in this paper, two mechanisms are involved, both connected to the presence of a nanometric electrical double layer on the surface of the colloid [1]: The first is purely mechanical and can be explained as a consequence of the existence of gradients of excess osmotic pressure inside the double layer, while the second is due to electrophoresis of particles in the electric field induced by the difference in mobility of positive and negative salt ions. Interestingly, both contributions lead to an additional transport term for the colloids of the same form, proportional to $\nabla \log S$ [1], where $S(\mathbf{x}, t)$ is the salt concentration at position $\mathbf{x}$ and time $t$; the total contribution is called the diffusiophoretic velocity, denoted by $\mathbf{v}_{\mathrm{dp}}$ [Eq. (3)]. The equations of motion are thus given by

$$
\begin{gathered}
\frac{\partial S}{\partial t}+\nabla \cdot[S \mathbf{v}]=D_{s} \nabla^{2} S, \\
\frac{\partial C}{\partial t}+\nabla \cdot\left[C\left(\mathbf{v}+\mathbf{v}_{\mathrm{dp}}\right)\right]=D_{c} \nabla^{2} C, \\
\mathbf{v}_{\mathrm{dp}}=D_{\mathrm{dp}} \nabla \log S,
\end{gathered}
$$

where $C(\mathbf{x}, t)$ is the colloidal concentration, $\mathbf{v}(\mathbf{x}, t)$ is the advecting velocity field, $D_{c}$ and $D_{s}$ are the diffusion coefficients of colloid and salt, respectively, and $D_{\mathrm{dp}}$ is the diffusiophoretic diffusivity. This set of equations is valid only if $\mathbf{v}$ is negligibly modified by the movement of the colloids (one-way coupling), i.e., if the colloidal concentration is not too large; this is the case here. From Eq. (2) it 
is clear that colloidal concentration is coupled to that of salt via the diffusiophoretic drift velocity, while the salt concentration evolves freely according to Eq. (1).

Deseigne et al. [3] have studied how diffusiophoresis affects chaotic mixing in a micromixer (the so-called staggered herringbone mixer [4], $200 \mu \mathrm{m}$ wide and $115 \mu \mathrm{m}$ high). Using a global characterization - the normalized standard deviation of concentration, a classical tool in mixing studies - they observed a diffusiophoretic effect that was interpreted in terms of effective diffusivity (or effective Péclet number). In Ref. [3], diffusiophoresis was acting at micron scales and the question remains whether diffusiophoretic effects extend to chaotic mixing at the macroscale: Will it be able to spread over all length scales or will it remain ineffectively confined at the nanoscale to microscale? This requires the investigation of possible scale-to-scale coupling: While chaotic advection affects all scales of the concentration field from the large scales of the macrocontainer down to the smallest ones, where diffusion is effective [5-12], what happens when it is combined with diffusiophoresis, a mechanism that originates at the nanoscale? In addition to the very existence of the effect, the quantification of its global impact on mixing also needs to be further investigated.

In order to answer these questions, we study diffusiophoresis in a chaotic mixer at the macroscale, that is, having dimensions larger than those of microsystems by 2 to 3 orders of magnitude (up to an overall scale of $5 \mathrm{~cm}$ ). Also, as noted in the abstract, rather than the decay of the standard deviation of concentration, which is a global parameter commonly employed in studies of mixing, we instead apply a set of refined characterizing analyses, using multiscale tools available from the turbulence community, such as concentration spectra (Sec. III A) and second and fourth moments of probability density functions (PDFs) of scalar gradients (Sec. III B). These more sophisticated tools allow us to perform a scale-by-scale analysis and thus study how all scales of the concentration field are affected by diffusiophoresis. Finally, after observing the propagation of diffusiophoretic effects up to the macroscale, we discuss the introduction of an effective Péclet number: Indeed, diffusiophoresis is related to compressible effects through the diffusiophoretic velocity, which is not divergence-free, as shown numerically in Ref. [13]. Thus, it has similarities to the preferential concentration of inertial particles in turbulent flows [14,15].

\section{DESCRIPTION OF THE EXPERIMENT}

\section{A. Experimental setup}

Mixing takes place in a horizontal square Hele-Shaw cell of length $L=50 \mathrm{~mm}$ and height $h=4 \mathrm{~mm}$, fitted with four inlets and outlets (Fig. 1). Each inlet or outlet is pressure driven using a flow controller (Fluigent, MFCS). The Hele-Shaw cell is initially filled with water (or salted water). At $t=0,0.2 \mathrm{ml}$ of a fluorescent solution (either dye or colloidal suspension) is introduced via inlet 1 into the Hele-Shaw cell using a syringe pump. The four inlets and outlets are then pressurized to 100 mbars and fluid motion is induced by successive pressurization and depressurization of the inlets and outlets; a movie showing the mixing process is provided as Supplemental Material [16]. Successive deformations of the concentration field are visualized using planar laser-induced fluorescence: A continuous laser (Coherent Genesis MX SLM-Series, $\lambda=488 \pm 3 \mathrm{~nm}$ ) coupled to a cylindrical lens forms a laser sheet with a typical thickness of the order of the cell height so that the whole volume of the cell is illuminated. The choice of such a thick laser sheet, rather than a thin one localized at the midheight of the cell, will be discussed at the end of Sec. II C.

The fluorescence signal is recorded with a 14-bit camera (Nikon D700, $4200 \times 2800$ pixels $^{2}$ ) whose lens (with a zoom of $105 \mathrm{~mm}$ ) is equipped with a band reject filter (notch $488 \pm 12 \mathrm{~nm}$ ) corresponding to the laser wavelength. The ISO sensitivity is set to the lowest value in order to avoid noise, the aperture is set to the highest possible (i.e., $f / 3.5$ ), with a shutter speed of $12.5 \mathrm{~ms}$. Image resolution in both horizontal directions ( $x$ or $y$ ) is about $19 \mu \mathrm{m} \mathrm{pixel}^{-1}$, while the depth of field is of the order of $1 \mathrm{~mm}$. Calibration for different fluorescent species and different concentrations showed a linear relationship between the light intensity and the concentration of the species throughout the range studied. 


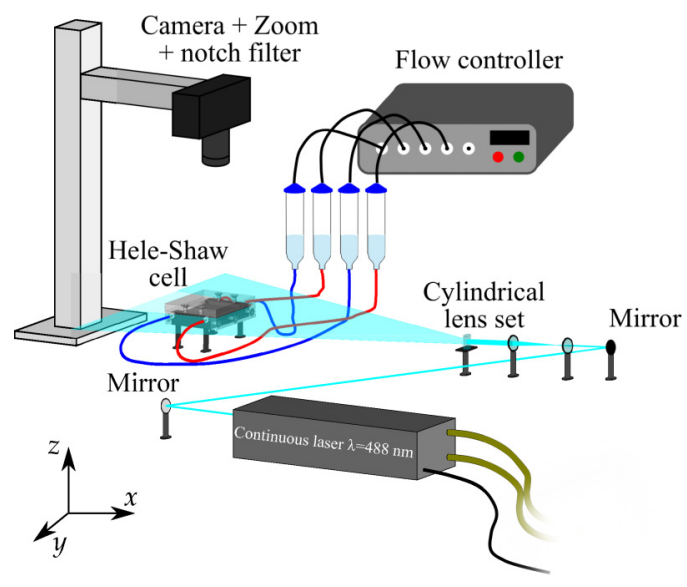

(a)

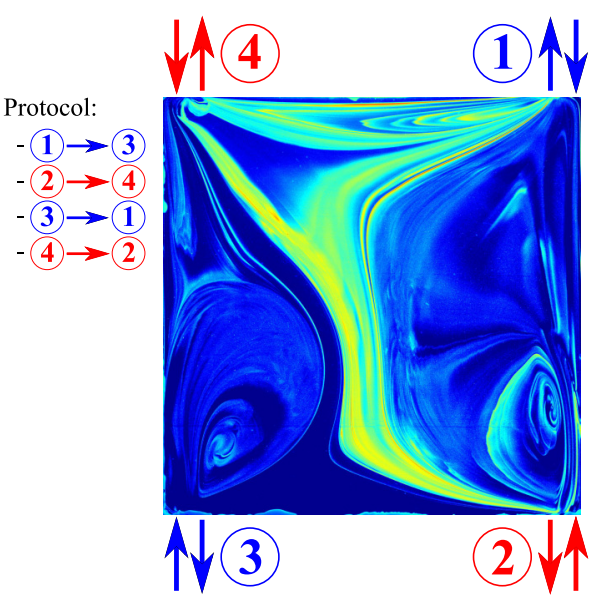

(b)

FIG. 1. (a) Scheme of the experimental setup: The square Hele-Shaw cell $(L=50 \mathrm{~mm})$ lies horizontally in the $x y$ plane. (b) Time-periodic mixing protocol: (i) $\rightarrow$ (i) indicates that at this step the fluid enters at (i) and exits at (i) during a lapse of time $T / 4$, with $T$ the period of the flow field. The figure displays the instantaneous pattern of a typical concentration field (colloids without salt). A movie showing the observed concentration patterns during the whole mixing process is also provided as Supplemental Material [16].

\section{B. Flow rate and mixing}

Chaotic advection is produced using the time-periodic protocol illustrated in Fig. 1(b), with four stages of duration $T / 4$. The efficiency of chaotic mixing in such a Hele-Shaw cell is qualified by the dimensionless pulse volume $\alpha$ :

$$
\alpha=\frac{q T}{L^{2} h}
$$

where $q$ is the flow rate and $\alpha$ represents the volume of fluid displaced during one period compared to the volume of the chamber $[17,18]$. For this particular mixing protocol, global chaos (no visible regular region) is obtained for $\alpha \geqslant 1.2$ [17,19]. Because large values of $\alpha$ imply rather high flow rates (hence large Reynolds numbers) or large periods $T$ (hence very long mixing time [20]), we chose to consider the smallest value of interest $\alpha=1.2$.

In a Hele-Shaw cell, the Reynolds number $\mathrm{Re}_{h}$ is conventionally based on the height $h$ of the cell, i.e., with typical velocity $q / h L$ and kinematic viscosity $v$,

$$
\operatorname{Re}_{h}=\frac{q}{L v} .
$$

Note that the Reynolds number inside the pipes connected to the inlets and outlets

$$
\operatorname{Re}_{\text {pipes }}=\frac{4 q}{\pi d \nu},
$$

with $d=1 \mathrm{~mm}$ the diameter of the pipes, is considerably higher. Because in the present case $\mathrm{Re}_{\text {pipes }}=64 \mathrm{Re}_{h}$, we set $\mathrm{Re}_{h}=1$ to avoid having too large a Reynolds number in the pipes and hence nonreproducible experiments. This corresponds to a flow rate $q=50 \mu \mathrm{L} \mathrm{s}^{-1}$ and, providing $\alpha=1.2$, we obtain the period $T=120 \mathrm{~s}$. Note that with those parameters, the flow is laminar and deterministic, as can also be appreciated in the movie [16]. As a consequence, the advecting velocity $\mathbf{v}$ in Eqs. (1) and (2) is identical for all the cases considered here (except for the short initial transient stratification, discussed in the Appendix for cases with salt). Each of the experiments in this article was carried out twice in order to verify that the indicators computed in Sec. III were reproducible. 
CYRIL MAUGER et al.

TABLE I. Species used, diffusion coefficients, and corresponding Péclet numbers.

\begin{tabular}{lcc}
\hline \hline Species & Diffusion coefficient $\left(\mathrm{m}^{2} \mathrm{~s}^{-1}\right)$ & Péclet number \\
\hline colloids & $2 \times 10^{-12}$ & $6 \times 10^{6}$ \\
dextran & $3.6 \times 10^{-11}$ & $3 \times 10^{5}$ \\
fluorescein & $4 . \times 10^{-10}$ & $3 \times 10^{4}$ \\
salt $(\mathrm{LiCl})$ & $1.4 \times 10^{-9}$ & $9 \times 10^{3}$ \\
\hline \hline
\end{tabular}

Since we are interested in mixing, the relevant parameter is the Péclet number, which measures the relative effect of advection compared to diffusion. Because in a Hele-Shaw flow chaotic mixing essentially takes place in the horizontal direction [21], we use the Péclet number based on the width $L$ of the cell,

$$
\mathrm{Pe}=\frac{q}{h D}
$$

where $D$ is the diffusion coefficient of the species considered.

For this study we used colloids of diameter $200 \mathrm{~nm}$ (FluoSpheres, LifeTechnologies F8811), marked with a yellow-green fluorophore (wavelength 505-515 nm). In order to characterize the efficiency of mixing as a function of the Péclet number (at fixed geometry and flow forcing), other species have also been used, namely, fluorescein isothiocyanate and fluorescent dextran $70000 \mathrm{MW}$ (LifeTechnologies D1823). For such molecular species diffusiophoresis is not expected to play a role; they are only used to quantify the deviations induced by diffusiophoresis in the case of colloids with salt. The diffusion coefficients and corresponding Péclet numbers for all species used in the experiment are available in Table I: The variation amplitude of the Péclet number is more than two orders of magnitude.

\section{Diffusiophoresis}

In order to induce diffusiophoresis, we used a $20 \mathrm{mM}$ solution of salt $(\mathrm{LiCl})$. Indeed, $\mathrm{LiCl}$ was shown in microfluidic experiments to have a stronger diffusiophoretic effect than other salts [2]: For these species (colloid and salt), the diffusiophoretic diffusivity is $D_{\mathrm{dp}}=290 \mu \mathrm{m}^{2} \mathrm{~s}^{-1}$ and the diffusiophoretic motion of the colloids goes from low- to high-salt-concentration regions [2].

In the following we discuss the interplay between mixing and diffusiophoretic drift by considering three different cases: the reference case, in which the colloids are injected into pure water; the salt-in case, in which the salt is introduced together with the colloids into pure water [in this configuration diffusiophoresis showed hypodiffusion (delayed mixing) in the staggered herringbone micromixer [3]]; and the salt-out case, in which the colloids are injected into salted water [in this configuration diffusiophoresis showed hyperdiffusion (enhanced mixing) in the staggered herringbone micromixer].

Recall from Eqs. (1) and (2) that, whereas the colloidal concentration is coupled to that of salt, the salt concentration $S$ freely evolves during the experiment. Thus, the salt is fully mixed for $t \geqslant L^{2} h \ln \left(\mathrm{Pe}_{s}\right) / 2 q[22,23]$, with $\mathrm{Pe}_{s}=q / h D_{s}$ the Péclet number for salt, that is, $t \sim 900 \mathrm{~s}$ with our parameters. After that time, diffusiophoresis no longer affects the colloids (although the global effect is still visible, i.e., mixing enhancement or reduction [13]). In what follows we will restrict attention to times where diffusiophoresis is fully effective.

Note finally that, because of buoyancy effects, the salt tends to rapidly stratify inside the cell (see the Appendix). Hence, although they have almost the same density as water, the colloids tend to flow from midheight, where they are injected, towards the bottom of the cell because of vertical diffusiophoresis induced by the salt concentration gradient (Appendix). This "settling" of colloids, which is only visible when salt is present and which goes against the effective buoyancy (more salted water in the bottom being denser than colloids), reveals one macroscopic effect of diffusiophoresis. 


\section{DIFFUSIOPHORESIS AT THE MACROSCALE}

Because it was difficult to follow the colloids over long times using a thin laser sheet (they would eventually disappear below the sheet) and because the flow is quasi-two-dimensional, we chose to illuminate the whole cell. This kind of height averaging can result in a loss of signal at small scales, especially in the salt-in case. Note that the coupling of the parabolic velocity profile with diffusion also leads to a vertical homogenization of the concentration field due to Taylor dispersion [24].

\section{RESULTS}

When measuring mixing efficiency, the quantity commonly used is the rate of decay of standard deviation of the concentration $C_{\text {std }}(t)=\left\langle(C-\langle C\rangle)^{2}\right\rangle^{1 / 2}$, or the nondimensional standard deviation $\sigma(t)=C_{\text {std }}(t) / C_{\text {std }}(t=0)$ [25], where $\langle\cdot\rangle$ stands for the spatial average. Indeed, without diffusiophoresis, the rate of decay of $C_{\text {std }}(t)$ is related to the presence of high scalar concentration gradients through the equation

$$
\frac{d C_{\mathrm{std}}^{2}}{d t}=-2 D\left\langle(\nabla C)^{2}\right\rangle .
$$

Note that diffusion operates at all scales, but is much more efficient at small scale where the gradients are more intense. In the following, as commonly done by fluid mechanics, the quantity $\frac{1}{2}(C-\langle C\rangle)^{2}=\frac{1}{2} C_{\text {std }}^{2}(t)$ is referred to as scalar energy, by analogy with the kinetic energy.

Above all, chaotic advection involves a large range of scalar scales from the macroscale of the experiment down to the smallest length scale involved, while diffusiophoresis involves a mechanism at the nanoscale. Thus such a global parameter as $\sigma$ is not enough to explore this typically multiscale coupled problem. For instance, does diffusiophoresis strongly dissipate scalar energy at a very small scale or does it interact with the flow so as to dissipate more smoothly at all length scales involved? In addition, let us note that even for a global characterization, $\sigma$ would not be an appropriate parameter here anyway since the flow is an open flow [marked particles go in and outside the chamber through the inlets and outlets during the periodic mixing protocol, i.e., $\langle C\rangle(t) \neq$ const].

In order to investigate the multiscale properties of the concentration field, we use different tools adapted for such a multiscale process. One is the scalar energy spectrum $E_{\theta}(k)$, which is commonly employed in chaotic advection studies [8,26-29]; it quantifies the scalar energy contained at a given wave number $k=2 \pi / \ell$, where $\ell$ can be seen as the physical scale at which the scalar energy is calculated, i.e., the typical width of a scalar structure; it is linked to the global scalar energy through the relation $\frac{1}{2} C_{\text {std }}^{2}=\int_{0}^{\infty} E_{\theta}(k) d k$. We also use PDFs of scalar gradients (more widely encountered in turbulent mixing [30,31]; see also [8,32]); while global dissipation of scalar energy is linked to concentration gradients through Eq. (8), such a distribution allows one to investigate whether dissipation occurs mainly with gradients quite close to the mean gradient (as can be seen, for instance, with a Gaussian distribution) or is related to very intense local gradients, in which case we refer to spatial intermittency. In the present study, each image (corresponding to a given time $t$ ) allows us to obtain $O\left(6 \times 10^{6}\right)$ values of the concentration gradient in each direction, further used to compute one PDF.

\section{A. Scalar energy spectra}

Instantaneous scalar energy spectra $E_{\theta}(k)$ are calculated from individual concentration fields at a given time by using the two-dimensional Fourier transform $\hat{\theta}\left(k_{x}, k_{y}, t\right)$ of the reduced concentration field $\theta(x, y, t)=[C(x, y, t)-\langle C(x, y, t)\rangle] / C_{\text {std }}(t)$; in order to reduce aliasing due to nonperiodic boundary conditions, a window Hanning method was used. The one-dimensional isotropic spectrum was then obtained by averaging over each $k=\left(k_{x}^{2}+k_{y}^{2}\right)^{1 / 2}$.

Figure 2(a) shows typical instantaneous scalar energy spectra, plotted on log-log scale, for the three configurations: the reference case (without salt), the salt-in case, and the salt-out case. Clearly, the small amount of salt visibly impacts the whole spectrum, although small scalar scales are more affected than large scales (as for diffusion effects). In the salt-in case (closed squares), the spectrum 


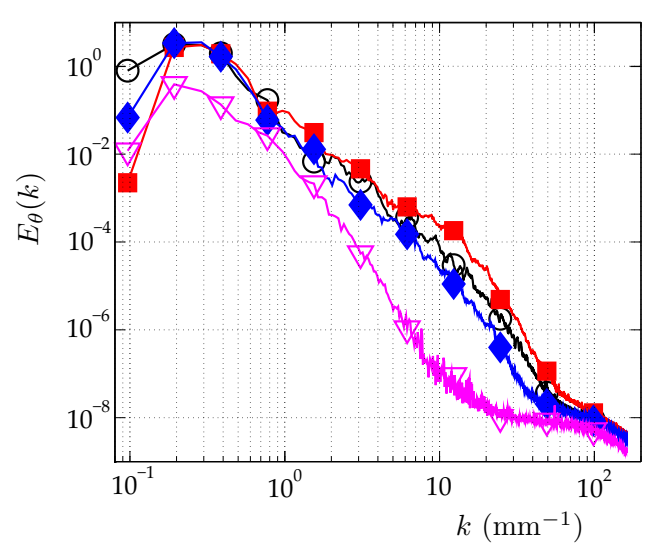

(a)

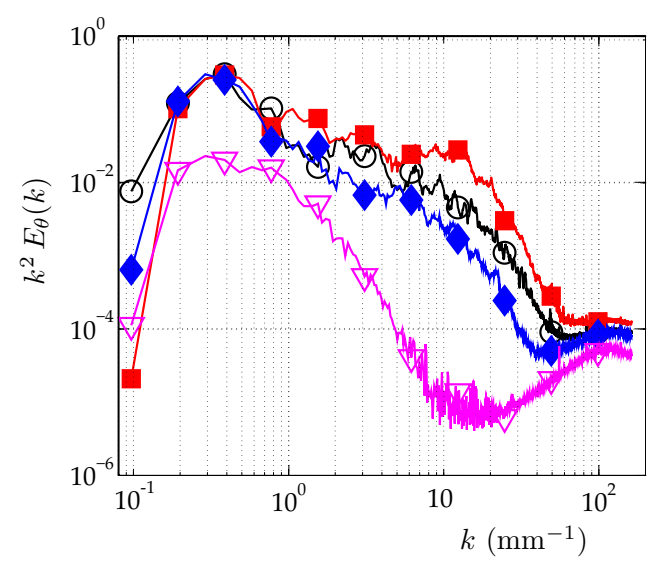

(b)

FIG. 2. (a) Instantaneous spectra of scalar energy (time $t=160 \mathrm{~s}$ ). (b) Instantaneous dissipation spectra for the same time. Open symbols stand for cases without salt; o, reference case (no salt); $\mathbf{\square}$, salt-in case; $\downarrow$, salt-out case; and $\nabla$, fluorescein. The scalar energy spectrum and dissipation spectrum for fluorescein have been divided by 10 .

extends further towards large wave numbers (small scales) than the reference spectrum. This kind of behavior would also be observed if considering the concentration spectrum of a species that diffuses less than the colloid we used. Indeed, since diffusion is directly related to scalar dissipation through Eq. (8), a smaller diffusion coefficient $D$ (therefore a larger Péclet number) implies that the final scalar dissipation occurs with larger concentration gradients, i.e., at an even smaller length scale: The spectrum would also be shifted towards larger wave numbers. In the salt-out case, the effect is reversed, with a shift towards smaller wave numbers. As a comparison and in order to show the influence of a much smaller Péclet number, we have also plotted in the figure the spectrum of fluorescein, although it was divided by 10 for clarity.

The effect is even clearer in Fig. 2(b) when looking at the term $k^{2} E_{\theta}(k)$, proportional to the scale by scale dissipation budget: Diffusiophoresis obviously affects all length scales ranging roughly from the centimeter $\left(k \geqslant 0.8 \mathrm{~mm}^{-1}\right)$ down to the smallest scales resolved. Quite remarkably, this demonstrates that diffusiophoresis can indeed influence mixing processes way beyond its nanometric roots or its micrometric classical influence. Combined with the chaotic mixing multiscale process, it can spread over more than seven orders of magnitude in length scales and affect the global system.

However, one should note that the previous diagnosis relies on an instantaneous analysis: While the flow is time periodic, the large-scale concentration patterns, and therefore the large scales of the associated spectra, also vary with time, as can be appreciated in the movie included as Supplemental Material [16]. Indeed, the effect is not always as pronounced as in Fig. 2; at some (rare) moments of the periodic cycle the effect is even reversed, as also found in our numerical simulations [13]. Because most of the scalar energy is contained in the largest scales (hence in the smallest wave numbers $k$ ) and because the large concentration scales vary with time, it is not easy to obtain from the spectra a time-averaged parameter that would accurately measure a global effect of salt. As observed in the spectra, small scalar scales are more affected by diffusiophoresis; we therefore propose to investigate the scalar gradients so as to obtain a quantitative comparison that considers a global effect over time.

\section{B. Concentration gradients}

In order to obtain the concentration gradients $\mathbf{G}=\nabla C$, a given image of the concentration field (corresponding to a given time $t$ ) is first filtered using a Gaussian kernel to get rid of potential noise: 


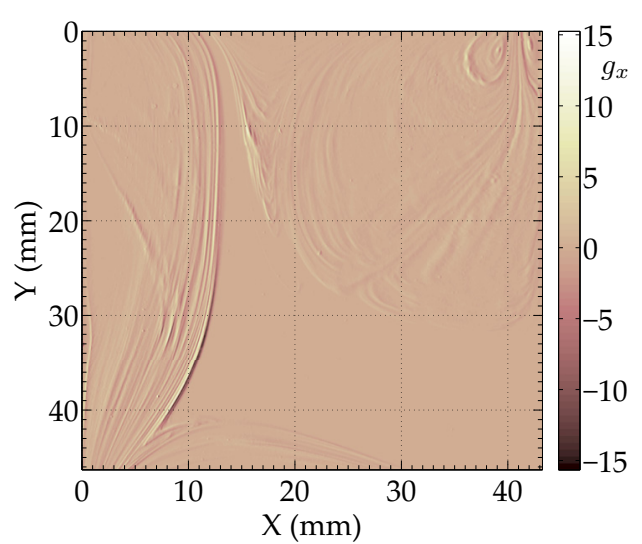

(a)

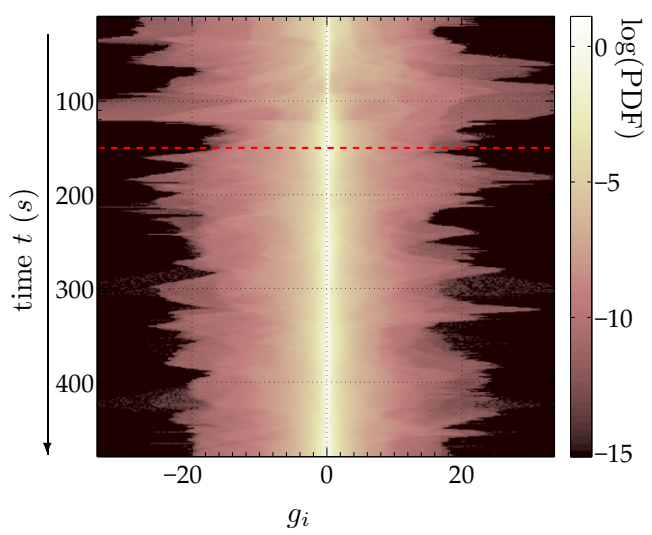

(b)

FIG. 3. Reference case with colloids (no salt). (a) Instantaneous reduced gradient $x$ component, with $g_{x}=\left(G_{x}-\left\langle G_{x}\right\rangle\right) / G_{x \mid \text { std }}$, where $G_{x}=\partial C / \partial x$ (at time $t=293 \mathrm{~s}$ ). (b) Time evolution of $\log (\mathrm{PDF})$ of all reduced gradient components $g_{i}$, where $g_{i}$ stands for $g_{x}$ and $g_{y}\left(\operatorname{PDF}\left(g_{i}\right)=1 / 2\left[\operatorname{PDF}\left(g_{x}\right)+\operatorname{PDF}\left(g_{y}\right)\right]\right)$. The dotted line at $t=150 \mathrm{~s}$ corresponds to the moment when PDFs become reasonably periodic in time, so that time averaging is conceivable.

Filtering over two pixels $(\approx 40 \mu \mathrm{m})$ is enough to obtain the gradients with great accuracy. Then we measure the two components of the concentration gradients, $G_{x}=\partial C / \partial x$ and $G_{y}=\partial C / \partial y$ at each point of the image. For component $x(y)$, we calculate the mean gradient component over the whole image $\left\langle G_{x}\right\rangle\left(\left\langle G_{y}\right\rangle\right)$ and also the standard deviation $G_{x \mid \text { std }}=\left\langle\left(G_{x}-\left\langle G_{x}\right\rangle\right)^{2}\right\rangle^{1 / 2}\left(G_{y \mid \text { std }}\right)$.

In the following we investigate the reduced gradient component $g_{i}$ :

$$
g_{i}=\left(G_{i}-\left\langle G_{i}\right\rangle\right) / G_{i \mid \mathrm{std}},
$$

where $i$ stands for $x$ and $y$. In Fig. 3(a) we plot the reduced gradient $x$ component $g_{x}$ at a given time ( $t=293 \mathrm{~s}$, which corresponds to $2 \frac{1}{4}$ periods of the flow field) in the reference case (no salt). Note the very-large-amplitude range from -15 to 15 , indicating that the spatial fluctuations of the scalar concentration gradient are not Gaussian (events of large amplitude are more likely to happen than in a Gaussian case, which is commonly referred to as spatial intermittency). This is reminiscent of the intense and intermittent concentration gradient fronts produced by the mixing process, which are captured when computing this quantity. This results in stretched PDFs of scalar gradient as it will be shown later in Fig. 4(b). While $g_{x}$ and $g_{y}$ have equivalent statistics, it is interesting to consider the mean statistics that are even better converged: Figure 3(b) shows the PDF of the normalized gradient component $g_{i}, \operatorname{PDF}\left(g_{i}\right)=1 / 2\left[\operatorname{PDF}\left(g_{x}\right)+\operatorname{PDF}\left(g_{y}\right)\right]$, as a function of time (one PDF every second). In the experiment, after a transient mixing phase where the initial spot of marked dye begins to spread in the whole domain (roughly one period of the flow field $T$ ), the global patterns become almost periodic with time (with period of the flow field), i.e., have a similar shape each period. This is also visible in Fig. 3(b) for times $t \geqslant 150 \mathrm{~s}$ (shown with a dotted line in the figure), where the PDFs have a similar shape every period $T=120 \mathrm{~s}$, with abrupt events occurring typically every $T / 4$, i.e., associated with a different phase of the periodic protocol [Fig. 1(b)]. In the following we consider time-averaged data, denoted by an overbar, averaged on the interval of time $150 \mathrm{~s} \leqslant t \leqslant 470 \mathrm{~s}$. We can now compare cases with or without salt.

For each image (i.e., for each time), we define the Taylor length scale associated with concentration gradients as $\lambda=2 C_{\text {std }} / \sqrt{G_{x \mid \text { std }}^{2}+G_{y \mid \text { std }}^{2}}$ and consider its time-average value $\bar{\lambda}$ (averaged over $150 \mathrm{~s} \leqslant t \leqslant 470 \mathrm{~s}$ ) in Fig. 4(a). When mixing without salt is considered (open symbols, corresponding to cases without any diffusiophoretic effect), $\bar{\lambda}$ roughly follows a decaying power law with Péclet number. In the salt-out case, $\bar{\lambda}$ is clearly greater than in the reference case. We 


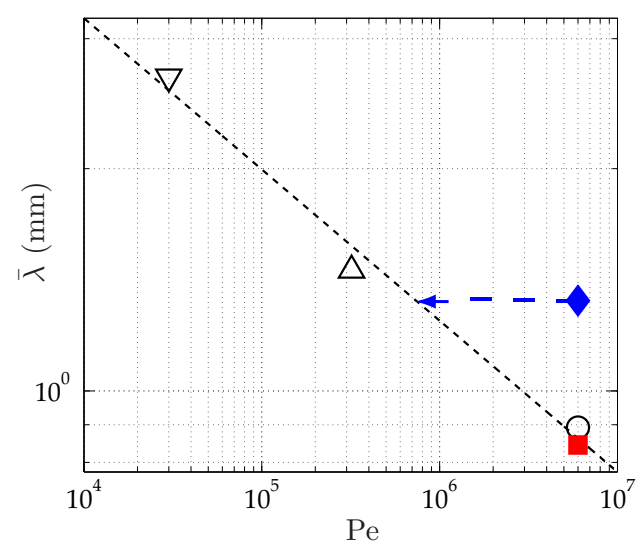

(a)

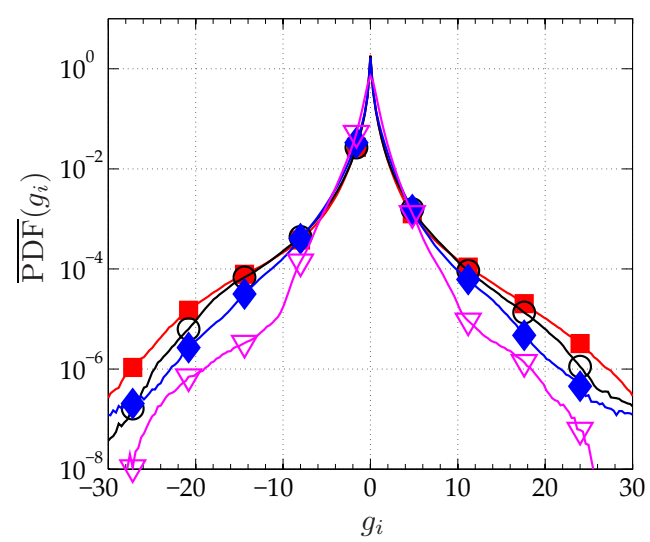

(b)

FIG. 4. (a) Taylor scale of scalar gradients $\bar{\lambda}$ defined as the time average of $\lambda=2 C_{\text {std }} / \sqrt{G_{x \mid \text { std }}^{2}+G_{y \mid \text { std }}^{2}}$. The arrow indicates the effective Péclet number defined as the corresponding reference Péclet number that leads to the same value of $\bar{\lambda}$. (b) Time-averaged PDF of reduced scalar gradients $g_{i}, \overline{\operatorname{PDF}}\left(g_{i}\right)$. Open symbols stand for cases without salt; $\circ$, reference case (no salt); $\mathbf{\square}$, salt-in case; $\diamond$, salt-out case; and $\nabla$, fluorescein. For the sake of clarity dextran is omitted in this plot.

can define an effective Péclet number as the corresponding reference Péclet number that leads to the same value of $\bar{\lambda}$ (as suggested by the arrow); we obtain a much smaller effective Péclet number than for the reference case, $\mathrm{Pe}_{\mathrm{eff}}^{\text {salt-out }} \approx 8 \times 10^{5}$, which has to be compared to $\mathrm{Pe} \sim 6 \times 10^{6}$. In the salt-in case, the effect is less clear; this may be due to the very definition of this quantity, only based on standard values of concentration and gradients (second-order statistics), which are not as sensitive to the intermittency of the concentration field as are higher-order moments. In order to check this hypothesis we plot in Fig. 4(b) the time average of the instantaneous $\operatorname{PDF}\left(g_{i}\right)$, denoted by $\overline{\operatorname{PDF}}\left(g_{i}\right)$, with or without salt; for the sake of clarity we omitted the plot for dextran. When first comparing the cases without salt (open symbols, corresponding to colloids and fluorescein mixing statistics), we recover the usual enhancement of small-scale scalar intermittency with increasing Pe [30]: The wings of the PDF, plotted on a semilogarithmic scale, are much higher, suggesting that events of large amplitude are more likely to happen. When salt is added (closed symbols), once again we recover (with a time-averaged plot rather than the instantaneous ones of Fig. 2) that the salt-out configuration corresponds globally to a smaller effective Péclet number. In the salt-in case, we observe the effect of a larger Péclet number for strong values of gradients, although the plot is hardly distinguishable from the reference case for $-15 \leqslant\left|g_{i}\right| \leqslant 15$ [which explains indeed why the two corresponding points are so close in Fig. 4(a)]. Because the effect of intermittency is more visible on the fourth moment than on the second one, we propose to calculate the flatness of this time-averaged distribution. Indeed, the quantity $g_{i}^{4} \overline{\mathrm{PDF}}\left(g_{i}\right)$, plotted in Fig. 5(a), shows a pronounced effect in the salt-in case. This is even more visible when considering the flatness $F$ of the distribution shown in Fig. 5(b): While the flatness in the cases without salt remarkably follows an increasing power-law with the Péclet number, the salt-out case rather corresponds to an effective Péclet number roughly the same as the one found with the second moment of gradients $\bar{\lambda}, \mathrm{Pe}_{\text {eff }}^{\text {saltout }} \approx 10^{6}\left(\mathrm{Pe}_{\text {eff }}^{\text {saltout }} / \mathrm{Pe} \sim 1 / 6\right)$, while the salt-in case leads to $\mathrm{Pe}_{\mathrm{eff}}^{\text {salt-in }} \approx 2 \times 10^{7}\left(\mathrm{Pe}_{\mathrm{eff}}^{\text {salt-in }} / \mathrm{Pe} \sim 3\right)$.

\section{Discussion}

Overall, our experimental results show that nanoscale diffusiophoresis affects large particles mixing at the macroscale. While the results were quantified above using an effective Péclet number, it must be kept clear that the underlying mechanism is not diffusion. Rather, it is related to compressible 


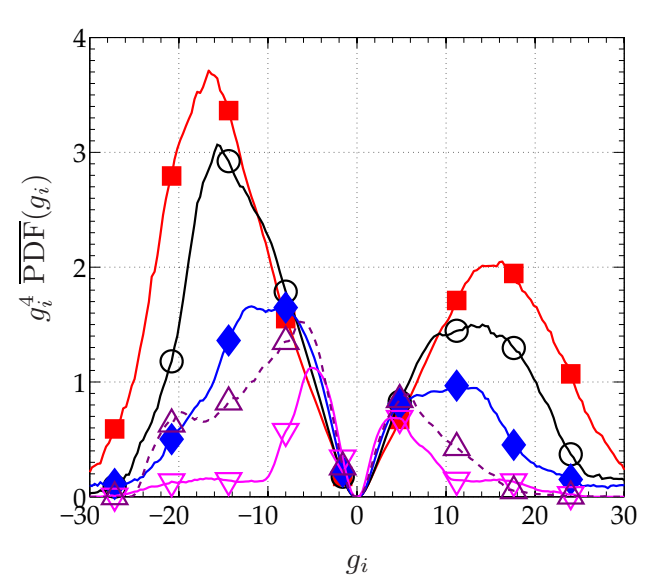

(a)

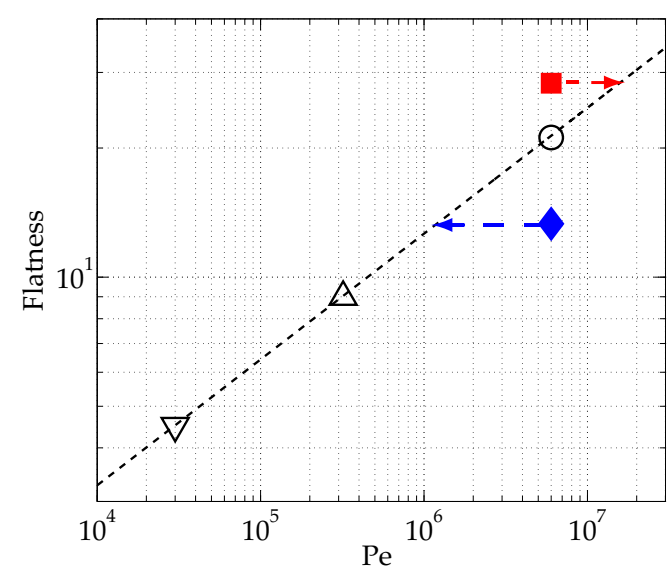

(b)

FIG. 5. (a) Quantity $g_{i}^{4} \overline{\mathrm{PDF}}\left(g_{i}\right)$, where $\overline{\mathrm{PDF}}\left(g_{i}\right)$ is the time-averaged PDF of $g_{i}$ for the cases under study. (b) Flatness of the time-averaged PDF $F=\left[\int g_{i}^{4} \overline{\mathrm{PDF}}\left(g_{i}\right) d g_{i}\right] /\left[\int g_{i}^{2} \overline{\mathrm{PDF}}\left(g_{i}\right) d g_{i}\right]^{2}$, with $\left\|g_{i}\right\| \leqslant 30$. The arrows indicate the effective Péclet numbers defined as the corresponding reference Péclet numbers that lead to the same flatness. Open symbols stand for cases without salt; $\circ$, reference case (no salt); $\boldsymbol{\square}$, salt-in case; $\downarrow$, salt-out case; $\triangle$, dextran; and $\nabla$, fluorescein.

effects through the diffusiophoretic velocity that is not divergence-free: $\nabla \cdot \mathbf{v}_{\mathrm{dp}}=D_{\mathrm{dp}} \nabla^{2} \log S$ is generally not zero in the presence of salt gradients. Thus, although the colloids are transported by the total velocity field $\mathbf{v}+\mathbf{v}_{\mathrm{dp}}$ [Eq. (2)], the effect is expected to be more complex than a large-scale effect through a large ratio of velocity amplitudes $V_{\mathrm{dp}} / V$. Indeed, using an order of magnitude estimate, one can prove this ratio to be less than $1 \%$ here: From Eq. (3) we obtain

$$
V_{\mathrm{dp}} \sim \frac{D_{\mathrm{dp}}}{\ell_{s}},
$$

where $\ell_{s}$ is the typical length scale of salt concentration gradients, which results from a competition of contraction by the chaotic flow field and diffusion. Because the salt is not coupled to the colloids, it obeys [22]

$$
\ell_{s} \sim \frac{L}{\sqrt{\mathrm{Pe}_{s}}},
$$

where $\mathrm{Pe}_{s}$ is the Péclet number of salt. Finally, from Eqs. (10) and (11) we obtain

$$
\frac{V_{\mathrm{dp}}}{V} \sim \frac{D_{\mathrm{dp}}}{\sqrt{D_{c} D_{s}}} \mathrm{Pe}^{-1 / 2} ;
$$

this order of magnitude is in accord with what we found numerically [13] [with the parameters used for our numerical study we obtain from Eq. (12) that $V_{\mathrm{dp}} / V \sim 4 \times 10^{-3}$ while we found numerically $6.5 \times 10^{-3}$ at $\mathrm{Pe}=6.5 \times 10^{4} \mathrm{]}$; in our experiment we obtain an even smaller ratio, $V_{\mathrm{dp}} / V \sim 2.2 \times 10^{-3}$.

Although useful and convenient, the effective Péclet approach is only approximate and is more appropriate in the salt-out configuration where mixing is enhanced. In that respect, it is quite remarkable that the effective Péclet for the salt-out case is indeed robust against the experimental observable used, either the Taylor scale of scalar gradients or the flatness of the distribution. In the salt-in case diffusiophoresis acts against diffusion, effectively inducing an "antidiffusion" that strengthens gradients at early times. Indeed, we have shown in our numerical work [13] that a global parameter such as the standard deviation of concentration $\sigma$ could increase at small times in the salt-in case, whereas diffusion can only cause $\sigma$ to decrease with time (8). This is the reason why salt-in 
TABLE II. Three cases where diffusiophoresis is combined with chaotic advection; for the numerical case and the micromixer, we used a Péclet number in the middle range of those investigated.

\begin{tabular}{lcccc}
\hline \hline Case & $\mathrm{Pe}$ & $D_{\mathrm{dp}} / \sqrt{D_{c} D_{s}}$ & $\mathrm{Pe}_{\text {eff }}^{\text {salt-out }} / \mathrm{Pe}$ & $\mathrm{Pe}_{\text {eff }}^{\text {saltin }} / \mathrm{Pe}$ \\
\hline sine flow (numerical) & $10^{4}$ & 1 & $1 / 2$ & $3 / 2$ \\
herringbone mixer (experimental) & $9 \times 10^{4}$ & 5.56 & $1 / 40$ & 20 \\
present experiment & $6 \times 10^{6}$ & 5.56 & $1 / 6$ & 3 \\
\hline \hline
\end{tabular}

characteristics are not easily observed in averaged quantities and require going to the fourth-order moment of the distribution of gradients, rather than the Taylor scale associated to the second moment. Finally, because diffusiophoresis is related to compressible effects, one could wonder if the use of an effective Péclet number is relevant here. One hint can be found in the concentration spectra in Fig. 2 (hence in the spatial structures of the concentration field): This multiscale approach shows that diffusiophoresis affects all scales of the concentration field, although small scales are even more affected. Because the same could be said for diffusion, the effect of diffusiophoresis on the concentration field has some similarities with diffusive effects. Another hint derives directly from Eq. (12): The relative transport by diffusiophoresis compared to that by the velocity field decreases with the Péclet number. This is also true for diffusion compared to advection from the very definition of the Péclet number. This provides a clue as to why it is useful and meaningful to introduce an effective Péclet number when considering the long-time effects and to try to quantify the combined effects of diffusiophoresis and diffusion with that effective approach.

In the following we collect results obtained with diffusiophoresis in chaotic advection with different velocity fields: the herringbone micromixer [3], the sine flow [13], and the present flow. Although the flow field in the herringbone micromixer is stationary and three dimensional, it may however be compared favorably with what can be expected in a two-dimensional time-periodic flow: Indeed, Stroock and McGraw [33] proposed an analytical model in which the cross section of the channel is treated as a lid-driven cavity flow; they showed that this model was able to reproduce the advection patterns that were observed experimentally in their flow, whose dimensions are about the same as in Ref. [3] (roughly $200 \mu \mathrm{m}$ wide, $100 \mu \mathrm{m}$ high). Here, because of the spatial periodicity in the axial direction, the corresponding coordinate plays the role of time. Correspondingly, the Péclet number in the micromixer has to be based on the cross-sectional velocity rather than on the axial velocity. With their model, Stroock and McGraw could also estimate the magnitude of the velocity $u_{\text {cross }}$ in the cross-sectional flow relative to the axial velocity $U$ : taking $u_{\text {cross }} \sim 0.1 U$, with a channel width $w=200 \mu \mathrm{m}$ and $U=8.6 \mathrm{~mm} / \mathrm{s}$, we obtain a colloidal Péclet number Pe $\sim 9 \times 10^{4}$.

All the results are summarized in Table II. In order to compare numerical and experimental results, we introduced the diffusiophoretic coefficient $D_{\mathrm{dp}}$ (equal to $290 \mu \mathrm{m}^{2} \mathrm{~s}^{-1}$ in both experiments) using a dimensionless parameter; because of Eq. (12), we chose to compare $D_{\mathrm{dp}} / \sqrt{D_{c} D_{s}}$.

It is not easy to compare those three cases: Not only are the Péclet numbers different, but also the diffusiophoretic coefficient is higher in the experiments. Note also that the present flow is an open flow $[\langle C\rangle(t) \neq$ const], while the others are not: For the micromixer, $\langle C\rangle(t)=$ const in all planes perpendicular to the axial direction and the sine flow uses periodic boundary conditions. However, in all cases, the effect is more important in the salt-out than in the salt-in case. Moreover, for the two experiments where the same colloids and salts were used, we obtain quite a remarkable result, i.e., $\mathrm{Pe}^{2} \simeq 2 \mathrm{Pe}_{\text {eff }}^{\text {saltout }} \mathrm{Pe}_{\text {eff }}^{\text {salt-in }}$.

\section{SUMMARY}

In this article we have studied experimentally the effects of diffusiophoresis on chaotic mixing of colloidal particles in a Hele-Shaw cell at the macroscale. We have compared three configurations, one without salt (reference case), one with salt with the colloids (salt-in case), and a third one 
where the salt is in the buffer (salt-out case). Rather than the decay of the standard deviation of concentration, we have used different multiscale tools such as concentration spectra and the second and fourth moments of the PDFs of scalar gradients, which allow for a scale-by-scale analysis; those tools are also available in open flows, when marked particles can go in and out of the domain under study.

Using scalar spectra, we have shown qualitatively that diffusiophoresis affects all scalar scales. This demonstrates that this mechanism at the nanoscale has an effect at the centimetric scale, i.e., seven orders of magnitude larger. Because the smallest scalar scales are more affected, this results in a change of spatial intermittency of the scalar field: Using the second and fourth moments of the PDFs of scalar gradients, we have been able to quantify globally the impact of diffusiophoresis on mixing at the macroscale. Although diffusiophoresis is clearly induced by compressibility effects, we have explained how the combined effects of diffusiophoresis and diffusion are consistent when averaging in time with the introduction of an effective Péclet number: The salt-in configuration corresponds to a larger effective Péclet number than the reference case and the opposite for the salt-out configuration. Because this results from a time-averaged study and not from an instantaneous diagnostic, this demonstrates that diffusiophoresis, a mechanism that originates at the nanoscale, has a quantitative effect on mixing at the macroscale.

\section{ACKNOWLEDGMENTS}

We are very grateful to Jean-Pierre Hulin and Laurent Talon for helpful discussions on gravity currents. This collaborative work was supported by the LABEX iMUST (Grant No. ANR-10LABX-0064) of Université de Lyon, within the program "Investissements d'Avenir" (Grant No. ANR-11-IDEX-0007) operated by the French National Research Agency.

\section{APPENDIX: ABSENCE OF GRAVITY CURRENTS, STRATIFICATION OF SALT, AND ASSOCIATED MIGRATION OF COLLOIDS}

It may be thought that given the density difference between pure and salted water, we could observe gravity currents inside the Hele-Shaw cell at the early stages of the time-periodic flow (before salt begins to mix due to chaotic advection); the arguments given in this appendix prove that this is not the case.

The first, and indirect, proof is that such an additional velocity field would lead to an enhancement of mixing in all cases, while both an enhancement (salt-out) and a suppression (salt-in) are observed. The second argument can be obtained from the experiment of Séon et al. [34], who studied the relative interpenetration of two fluids of different densities in a nearly horizontal configuration. While their flow takes place in a tube rather than a Hele-Shaw cell, they consider fluids of the same viscosity, just like in the present experiment. The Atwood number in their case, At $=\left(\rho_{2}-\rho_{1}\right) /\left(\rho_{2}+\rho_{1}\right)$, where $\rho_{1}$ and $\rho_{2}$ are the densities of the fluids, ranges from $10^{-3}$ to $4 \times 10^{-2}$. In our case At $=$ $\triangle \rho / 2 \rho=\beta \triangle S / 2$, where $\rho$ is density and $\beta$ is the expansion coefficient; with $\beta=2.4 \times 10^{-2} \mathrm{M}^{-1}$ for $\mathrm{LiCl}$ [35] and $\triangle S=20 \mathrm{mM}$, we obtain At $\sim 2.4 \times 10^{-4}$, which makes our configuration more stable from this point of view. In the particular case of a perfectly horizontal tube, they obtain a decelerating front, whose initial speed is based on the viscous scales, that stops after some time. In our experiment, because of the vertical parabolic profile of the Hele-Shaw flow, such a velocity $v_{v}$ would scale like $\Delta \rho g \sim 12 \mu v_{v} / h^{2}$ (where $g$ is gravity), i.e., $v_{v} \sim \beta \Delta S g h^{2} / 12 v \sim 6 \mathrm{~mm} \mathrm{~s}^{-1}$, superimposed on the pressure-driven basic flow. While the mean velocity of the front in the reference case is of order $2 \mathrm{~mm} \mathrm{~s}^{-1}$, this phenomenon (even if transitory) would lead to a velocity three times higher, which would significantly change the positions of the fronts between the reference case and the salt-in or salt-out case; however, we did not observe any shift in the positions of the front between those three configurations.

The reason may be found in an article by Talon et al. [36]: In their computational paper, the flow takes place in a Hele-Shaw cell with a mean flow, like in our experiment, and fluids with different 
density and viscosity are considered. Because of gravity, they observe that the displacement front experiences a transitory state of higher velocity before reaching its stationary value; however, when the gravity parameter $F=\triangle \rho g h^{2} / \mu U$, which measures gravity versus viscous forces, is decreased towards unity, the transitory state disappears. In our experiment this parameter, based on the velocity at the entrance of the chamber, is of order unity. Thus the flow field in the three configurations (salt-in, reference, and salt-out cases) can be considered as identical, except for the density differences.

Past the early stages, the displacement front is stretched and folded by chaotic advection, which causes salt to begin to mix and stratify through a competition between gravity and diffusion: A vertical gradient of salt appears, which can settle a colloidal movement because of diffusiophoresis. Following Eq. (3), the vertical diffusiophoretic velocity $\mathbf{v}_{\mathrm{dp}}$ is of order $D_{\mathrm{dp}} \nabla S / S \sim D_{\mathrm{dp}} / h$. The typical time scale $\tau_{\mathrm{dp}}^{\mathrm{vert}}$ associated with vertical diffusiophoresis is the time taken for a particle to go from half depth where it is injected down to the bottom, hence $\tau_{\mathrm{dp}}^{\mathrm{vert}} \sim h^{2} / 2 D_{\mathrm{dp}} \sim 8 \mathrm{~h}$. Although this is rather long, we could observe, when using a very thin laser sheet (300 $\mu \mathrm{m}$ thick), that colloids tend to disappear below the sheet at large times in places of high stretching and folding rate. This is why we chose to illuminate the whole cell.

[1] J. L. Anderson, Colloid transport by interfacial forces, Annu. Rev. Fluid. Mech. 21, 61 (1989).

[2] B. Abécassis, C. Cottin-Bizonne, C. Ybert, A. Ajdari, and L. Bocquet, Osmotic manipulation of particles for microfluidic applications, New J. Phys. 11, 075022 (2009).

[3] J. Deseigne, C. Cottin-Bizonne, A. D. Stroock, L. Bocquet, and C. Ybert, How a "pinch of salt" can tune chaotic mixing of colloidal suspensions, Soft Matter 10, 4795 (2014).

[4] A. D. Stroock, S. K. W. Dertinger, A. Ajdari, I. Mezic, H. A. Stone, and G. M. Whitesides, Chaotic mixer for microchannels, Science 295, 647 (2002).

[5] H. Aref, Stirring by chaotic advection, J. Fluid Mech. 143, 1 (1984).

[6] J. M. Ottino, The Kinematics of Mixing: Stretching, Chaos and Transport (Cambridge University Press, New York, 1989).

[7] V. Rom-Kedar, A. Leonard, and S. Wiggins, An analytical study of the transport, mixing and chaos in an unsteady vortical flow, J. Fluid Mech. 214, 347 (1990).

[8] R. T. Pierrehumbert, Tracer microstructure in the large-eddy dominated regime, Chaos Solitons Fractals 4, 1091 (1994).

[9] S. Cerbelli, A. Adrover, and M. Giona, Enhanced diffusion regimes in bounded chaotic flows, Phys. Lett. A 312, 355 (2003).

[10] E. Gouillart, J.-L. Thiffeault, and M. D. Finn, Topological mixing with ghost rods, Phys. Rev. E 73, 036311 (2006).

[11] D. R. Lester, G. Metcalfe, and M. G. Trefry, Is Chaotic Advection Inherent to Porous Media Flow? Phys. Rev. Lett. 111, 174101 (2013).

[12] O. Gorodetskyi, M. F. M. Speetjens, and P. D. Anderson, Eigenmode analysis of advective-diffusive transport by the compact mapping method, Eur. J. Mech. B 49, 11 (2015).

[13] R. Volk, C. Mauger, M. Bourgoin, C. Cottin-Bizonne, C. Ybert, and F. Raynal, Chaotic mixing in effective compressible flows, Phys. Rev. E 90, 013027 (2014).

[14] M. Maxey, The gravitational settling of aerosol-particles in homogeneous turbulence and random flowfields, J. Fluid Mech. 174, 441 (1987).

[15] R. A. Shaw, Particle-turbulence interactions in atmospheric clouds, Annu. Rev. Fluid Mech. 35, 183 (2003).

[16] See Supplemental Material at http://link.aps.org/supplemental/10.1103/PhysRevFluids.1.034001 for a movie showing the type of concentration patterns observed in the time-periodic flow field $(T=120 \mathrm{~s})$, here in the reference case (colloids without salt).

[17] F. Raynal, A. Beuf, F. Plaza, J. Scott, P. Carrière, M. Cabrera, J.-P. Cloarec, and E. Souteyrand, Towards better DNA chip hybridization using chaotic advection, Phys. Fluids 19, 017112 (2007). 


\section{DIFFUSIOPHORESIS AT THE MACROSCALE}

[18] A. Beuf, J. N. Gence, P. Carrière, and F. Raynal, Chaotic mixing efficiency in different geometries of Hele-Shaw cells, Int. J. Heat Mass Transfer 53, 684 (2010).

[19] F. Raynal, F. Plaza, A. Beuf, P. Carrière, E. Souteyrand, J.-R. Martin, J.-P. Cloarec, and M. Cabrera, Study of a chaotic mixing system for DNA chip hybridization chambers, Phys. Fluids 16, L63 (2004).

[20] F. Raynal and J.-N. Gence, Efficient stirring in planar, time-periodic laminar flows, Chem. Eng. Sci. 50, 631 (1995).

[21] F. Raynal, A. Beuf, and P. Carrière, Numerical modeling of DNA-chip hybridization with chaotic advection, Biomicrofluidics 7, 034107 (2013).

[22] F. Raynal and J.-N. Gence, Energy saving in chaotic laminar mixing, Int. J. Heat Mass Transfer 40, 3267 (1997).

[23] E. Villermaux, A. D. Stroock, and H. A. Stone, Bridging kinematics and concentration content in a chaotic micromixer, Phys. Rev. E 77, 015301 (2008).

[24] G. I. Taylor, Dispersion of soluble matter in solvent flowing slowly through a tube, Proc. R. Soc. London Ser. A 219, 186 (1953).

[25] Handbook of Industrial Mixing: Science and Practice, edited by E. L. Paul, V. A. Atiemo-obeng, and S. M. Kresta (Wiley-Interscience, Hoboken, 2003).

[26] B. S. Williams, D. Marteau, and J. P. Gollub, Mixing of a passive scalar in magnetically forced twodimensional turbulence, Phys. Fluids 9, 2061 (1997).

[27] V. Toussaint, Ph. Carrière, J. Scott, and J.-N. Gence, Spectral decay of a passive scalar in chaotic mixing, Phys. Fluids 12, 2834 (2000).

[28] M.-C. Jullien, P. Castiglione, and P. Tabeling, Experimental Observation of Batchelor Dispersion of Passive Tracers, Phys. Rev. Lett. 85, 3636 (2000).

[29] P. Meunier and E. Villermaux, The diffusive strip method for scalar mixing in two dimensions, J. Fluid Mech. 662, 134 (2010).

[30] M. Holzer and E. D. Siggia, Turbulent mixing of a passive scalar, Phys. Fluids 6, 1820 (1994).

[31] Z. Warhaft, Passive scalars in turbulent flows, Annu. Rev. Fluid Mech. 32, 203 (2000).

[32] R. T. Pierrehumbert, Lattice models of advection-diffusion, Chaos 10, 61 (2000).

[33] A. D. Stroock and G. J. McGraw, Investigation of the staggered herringbone mixer with a simple analytical model, Philos. Trans. R. Soc. London A 362, 971 (2004).

[34] T. Séon, J. Znaien, D. Salin, J.-P. Hulin, E. J. Hinch, and B. Perrin, Transient buoyancy-driven front dynamics in nearly horizontal tubes, Phys. Fluids 19, 123603 (2007).

[35] W. M. Haynes (ed.), CRC Handbook of Chemistry and Physics, 92nd ed. (CRC, Boca Raton, 2011).

[36] L. Talon, N. Goyal, and E. Meiburg, Variable density and viscosity, miscible displacements in horizontal Hele-Shaw cells. Part 1. Linear stability analysis, J. Fluid Mech. 721, 268 (2013). 